\title{
Proceso de cuidado durante la gestación y el puerperio en mujeres de la zona rural de Caldas, Colombia. Una mirada desde lo cotidiano ${ }^{1}$
}

Healthcare during pregnancy and post partum among women of a rural area in Caldas, Colombia. A perspective from everyday life

Processo de cuidado durante a gestação e o puerpério em mulheres da zona rural de Caldas, Colômbia. Um olhar a partir do cotidiano

\author{
Vanesa Giraldo Gartner ${ }^{2}$
}

Recibido: 15 de septiembre 2018 • Enviado para modificación: 16 de junio 2019 • Aceptado: 13 de septiembre 2019 Giraldo-Gartner, V. (2019). Proceso de cuidado durante la gestación y el puerperio en mujeres de la zona rural de Caldas, Colombia. Una mirada desde lo cotidiano. Revista Ocupación Humana, 19 (1), 22-36. doi: https://doi. org/10.25214/25907816.271

\begin{abstract}
RESUMEN
Esta investigación tuvo como objetivo describir y analizar el proceso de cuidado durante la gestación y puerperio en una comunidad rural cafetera de Colombia. Se trata de un estudio de corte cualitativo-etnográfico, que incluyó jornadas de observación participante en ámbitos domiciliarios y hospitalarios, y entrevistas semiestructuradas y a profundidad. Participaron 45 mujeres de la comunidad, tres promotoras de salud, dos enfermeras jefe y dos médicos. Este trabajo reveló que el cuidado de las mujeres durante el embarazo y el posparto está guiado por la lógica andina de frío-caliente, que se imparte desde el ámbito doméstico y comunitario; media la asistencia de promotoras de salud, la atención hospitalaria y, esporádicamente, la intervención de terapeutas no biomédicos. Los análisis etnográficos contribuyen a los enfoques comunitarios en Terapia Ocupacional, mediante el estudio de las experiencias cotidianas de los sujetos en contextos socioculturales específicos.
\end{abstract}

\section{PALABRAS CLAVE}

embarazo, población rural, cultura

\footnotetext{
${ }^{1}$ Este artículo se deriva de la tesis titulada Caminos de montaña y pasillos de hospital. Gestación, parto y puerperio en mujeres campesinas de Palestina, Caldas, Colombia, realizada por la autora bajo la dirección del profesor César Abadía, para optar al título de Magíster en Salud Pública de la Universidad Nacional de Colombia.

${ }^{2}$ Antropóloga. Magíster en Salud Pública. Candidata a doctora en Antropología. Universidad de Massachusetts. Amherst, Massachusetts, Estados Unidos de América vgiraldoga@gmail.com. iD ORCID: https://orcid.org/0000-0001-5248-5933
} 


\begin{abstract}
The main goal of this work is to describe and analyze the healthcare process during pregnancy and postpartum in a rural coffee community of Colombia. It is a qualitative-ethnographic study that included observational sessions in domestic and hospital settings and semi-structured and in-depth interviews. Participants comprised 45 women from the community, three health promoters, two chief nurses and two doctors. This work revealed that the care of women during pregnancy and postpartum is guided by the Andean logic with regard to cold and hot that is taught in the domestic and community settings and is mediated by the assistance of healthcare promoters, hospital care and, occasionally, the intervention of non-biomedical therapists. Ethnographic analyzes contribute to community approaches in occupational therapy by studying the daily experiences of subjects in specific socio-cultural contexts.
\end{abstract}

\title{
KEY WORDS
}

pregnancy, rural population, culture

\section{RESUMO}

Esta pesquisa teve como objetivo descrever e analisar o processo de cuidado durante a gestação e puerpério em uma comunidade rural cafeeira da Colômbia. Trata-se de um estudo de cunho etnográfico-qualitativo que incluiu jornadas de observação, realizadas em domicílios e hospitais, bem como entrevistas semi-estruturadas e em profundidade. Participaram 45 mulheres da comunidade, três promotoras de saúde, duas enfermeiras-chefe e dois médicos. Este trabalho revelou que o cuidado das mulheres, durante a gravidez e o pós-parto, estão guiados pela lógica andina de "frio-quente", realizada desde o âmbito doméstico e comunitário; pela assistência das promotoras de saúde; pela atenção hospitalar; e, esporadicamente, pela intervenção de terapeutas não biomédicos. As análises etnográficas contribuem para os enfoques comunitários em Terapia Ocupacional, mediante o estudo das experiências cotidianas dos sujeitos em contextos socioculturais específicos.

\section{PALAVRAS-CHAVE}

gravidez, população rural, cultura

\section{Introducción}

La gestación es un hecho social que abarca una serie de conocimientos, prácticas y representaciones sociales en torno a la maternidad, el cuerpo y la vida. Está determinado por condiciones políticas, económicas y socioculturales (Ginsburg \& Rapp, 1995; Blázquez, 2005; Morgan \& Roberts, 2012), bajo las cuales interactúan diferentes elementos terapéuticos y mecanismos de atención que establecen un modo particular de cuidar de la mujer gestante.

La salud materna es, desde la llamada Década de la Naciones Unidas para la Mujer (1976-1986), una de las principales preocupaciones sobre la salud femenina a nivel global. Acorde con esta prioridad y las directrices de los Objetivos del Milenio, la Política Nacional de Salud Sexual y Reproductiva en Colombia, y sus versiones en los distintos departamentos, se ha 
priorizado en las últimas décadas la línea de "maternidad segura", que se apoya en el marco normativo general de las acciones en salud pública. Esto ha implicado un despliegue institucional hacia la población de mujeres gestantes que necesariamente se ancla a procesos locales de cuidado y representaciones particulares sobre la vida, la maternidad y la corporalidad femenina.

Algunos estudios han mostrado los aportes de terapeutas ocupacionales en la promoción de la salud de las gestantes (Arellano \& Vega, 2005; Fernandes, 2018; Rubio-Grillo, Perdomo-Oliver \& Zamudio-Espinosa, 2018), el acompañamiento en el parto y puerperio (Trovatti \& Pérez-Martínez, 2012), la promoción de la lactancia materna (Visser et.al., 2016), la preparación para la maternidad entendida como una ocupación (Acharya, 2014) y la reciprocidad en la interacción materno-infantil (Gee et al., 2017). Partiendo de la pregunta sobre la manera en que los saberes y las prácticas cotidianas afectan la salud (Rodríguez Hernández, Flores Martos, \& Moruno Miralles, 2012; Trovatti \& Peréz- Martínez, 2012; García-Ruiz, 2017), la Terapia Ocupacional hace contribuciones valiosas al establecimiento $y$ el mantenimiento de prácticas de cuidado de la salud materna como experiencia vital.

Desde una mirada etnográfica, este artículo describe y analiza el proceso de cuidado durante la gestación y el puerperio en una comunidad rural cafetera de Colombia. Se enfoca, en particular, en lo que emergió como principal criterio de bienestar del cuerpo de las gestantes: el equilibrio entre frío y caliente, dos propiedades del cuerpo y del medio ambiente que hacen parte del mundo de sentido alrededor de la maternidad.

\section{Métodos}

Se trata de un estudio de corte cualitativo-etnográfico desarrollado en la zona rural de Palestina, Caldas, en las veredas El Higuerón, La Plata, El Reposo, Cartagena, La Paloma y La Ermita. Esta región tiene una larga de tradición cafetera, lo que implica que recibió una fuerte influencia de las campañas de salud pública apoyadas por el gremio cafetero, que buscaban mejorar las condiciones de vida de la mano de obra dedicada a la economía del café. Esto la hace un lugar ideal para estudiar los efectos de las interacciones entre saberes populares y saberes biomédicos.

La recolección de datos consistió en jornadas de observación participante en espacios domésticos, puestos de salud y centros hospitalarios; registro en guías de observación; entrevistas semi-estructuradas, y entrevistas a profundidad.

Participaron 45 mujeres entre los 15 y los 54 años. Fueron seleccionadas mujeres gestantes, lactantes y cuidadoras que se reconocían como conocedoras de los cuidados durante la gestación, el parto y el puerperio. Se excluyeron aquellas mujeres que llevaban menos de un año en la región. Adicionalmente, participaron tres promotoras de salud, dos enfermeras jefe y dos médicos. La muestra se seleccionó de manera intencionada, hasta llegar a punto de saturación. La información obtenida mediante observación participante, entrevistas semi-estructuradas y entrevistas a profun- 
didad fue triangulada para validar los resultados de esta investigación.

El análisis de la información se realizó mediante categorías temáticas pre-determinadas y categorías emergentes que surgieron de las experiencias de las mujeres. Las primeras referían a redes de apoyo, cambios en las actividades cotidianas, cuidados caseros, relación con las promotoras de salud y acceso a la atención hospitalaria. Las dos categorías emergentes de mayor relevancia fueron la percepción de frío y caliente durante la gestación y el puerperio, y la interacción entre saberes locales y saberes biomédicos en los espacios hospitalarios. Para el análisis de los datos se empleó la herramienta informática de análisis cualitativo Atlas Ti 5.0.

En concordancia con las categorías mencionadas, los resultados se presentan siguiendo cuatro ejes relacionados con la experiencia de las mujeres: ámbito doméstico, promotoras de salud, atención hospitalaria y retorno al ámbito doméstico en el posparto.

\section{Consideraciones éticas}

Los lineamientos éticos de esta investigación se formularon de conformidad con la resolución 8430 de 1993 del Ministerio de Salud colombiano, por la cual se establecen las normas científicas, técnicas y administrativas para la investigación en salud. De acuerdo con el artículo 11 de dicha resolución, esta investigación se considera sin riesgo, dado que no se realiza ninguna intervención o modificación intencionada de las variables biológicas, fisiológicas, sicológicas o sociales de las personas que participaron en ella. Siguiendo el artículo 16, en su parágrafo primero, se incluyó el con- sentimiento informado verbal, consignado en las grabaciones de las entrevistas. Siguiendo los principios de confidencialidad en la investigación con seres humanos, este artículo no revela los nombres reales de los participantes.

El protocolo fue aprobado por el Consejo de la Facultad de Medicina de la Universidad Nacional de Colombia y por el Departamento Administrativo de Ciencia Tecnología e Innovación - Colciencias. Adicionalmente, obtuvo financiación de esta última entidad, a través del programa de Jóvenes Investigadores.

\section{Resultados}

\section{Ámbito doméstico comunitario}

En este trabajo, el ámbito doméstico y comunitario se entiende como la esfera social en la que confluyen distintas actividades ligadas al mantenimiento y cuidado de un grupo social. Allí coinciden las relaciones de parentesco, amistad y vecindad.

Equilibrio entre frío y caliente. Una de las categorías emergentes estuvo relacionada con la percepción del cuerpo de la mujer en equilibrio entre lo frío y lo caliente, como dos cualidades que no se refieren estrictamente a la temperatura corporal, sino a propiedades de los alimentos, las plantas y el medio ambiente. De acuerdo con ello, se considera que los cuerpos fríos, que perdieron calor o que no se están calentando lo suficiente son infértiles, carecen de fortaleza para concebir hijos sanos y sufren de dolores durante la gestación, el parto y el puerperio. No obstante, el exceso de calor también puede traer efectos nocivos para el feto y la madre. Las no- 
ciones de frío y caliente determinan las prácticas de cuidado perinatal en tanto a alimentación, actividades físicas y terapias caseras; asimismo, son un marco de interpretación sobre el funcionamiento de los métodos anticonceptivos.

Una amiga mía lleva un año buscando y nada, pero yo le dije que se esperara a desintoxicarse de las pastas; pero que eso sí, que tomara muchas cosas calientes, porque hay veces que la mujer es muy fría y hay unas que tienen la matriz muy descolgada y eso ayuda a que la mujer no quede en embarazo. Hay mujeres que en verdad de nacimiento son frías y eso no es culpa de la mujer, y la pasta (anticonceptivos) de todas maneras enfría harto. (Estela)

Para ello, las mujeres se comparten recetas de baños, alimentos y ungüentos que permiten calentar el cuerpo y lograr así el equilibrio que se requiere para gestar un bebé.

Compra viril de toro en un matadero, manda a que le quiten todos esos gordos, lo manda a pitar ${ }^{3}$ y a lo que ya esté pitado lo deja enfriar y le saca todos esos cueros y lo bota y le saca toda la grasa. Al caldo le masca un pitico de nuez moscada, le echa dos gelatinas de pata, media pastica de chocolate Luker, lo pone a hervir, lo bate bien batido, le echa un pitico de miel y se lo toma por ahí dos veces al mes; con eso si tiene bebé ligero y no es fría pa' estar con el esposo. (Julieta)
Cada uno de los ingredientes de esta receta es considerado caliente, por tanto, se le atribuye la capacidad de aumentar la fertilidad.

Alimentación y medio ambiente. La alimentación juega un rol fundamental en el mantenimiento del equilibrio entre frío y caliente. Las restricciones alimenticias durante el embarazo son relativamente pocas, se desaconsejan algunas frutas ácidas y las comidas frías o demasiado calientes que puedan afectar a la mujer o al feto. La clasificación entre alimentos fríos y calientes es un conocimiento que se hereda generacionalmente $y$, pese a ciertas diferencias entre familias, hay algunos consensos; por ejemplo, que la gallina, la canela y el comino son calientes, mientras que el pollo y la yuca son fríos. En ocasiones, se adicionan a la dieta coladas de maíz o plátano, suplementos nutricionales para niños y bebidas de cebada con leche. Quienes asisten a los controles prenatales en el hospital incluyen suplementos vitamínicos, si se los proporciona la institución.

Es bueno comer mucha fruta, mucha ensalada para que el bebé salga bien alentado. Es mejor no comer mucho limón o mango biche, pues que uno de pronto se antoje sí, pero cogerlo por costumbre $y$ comer y comer de eso no, le hace daño a ella y le hace daño al bebé, porque le puede picar anemia al bebé. Eso es cosa dañina. Con el niño mío yo me antoje de comer [...] cominos, ya me sabía la boca a cominos, iay qué ricura! Y una vez el marido me dijo: "mija ¿usted que

${ }^{3}$ Hervir en una olla a presión. 
está comiendo?" y yo le dije: "mijo es que es una ansiedad de comer cominos" y me dijo: "mija, pues es un antojo que usted tiene yo no le quitó la razón, iqué pecao!, pero le cuento que eso le hace daño al bebé, el bebé le puede nacer quemado". Le cuento que como a los cuatro días nació el niño, jay qué pecao mi bebé!, que usted viera esos jarreticos quemaditos, quemaditos, y fue el comino. "Si ve mija, donde yo no le diga usted sigue comiendo y sale el niño peor". (Mariela)

Los antojos deben cumplirse, si no quiere correrse el riesgo de que el bebé nazca con los labios entreabiertos como señal de frustración por no haber recibido lo que necesitaba, pero se debe tener mesura si se trata de un alimento peligroso. Además del tipo de alimentos, una precaución importante es no consumir preparaciones que hayan sido expuestas a los fríos nocturnos.

No puede comer uno comidas trasnochadas o cosas muy recalentadas. Todo como del mismo día. [...] cuidarse uno de no asolearse, no enserenarse, no lloviznarse $y$ comer muy bien. (Ligia)

Así como los alimentos, las condiciones climáticas, la luna llena y los eclipses pueden enfriar el cuerpo de la gestante. Como aporte a este equilibrio, los baños y las bebidas juegan un papel fundamental. Los baños consisten en infusiones de ramas calientes como brevo, arracacha, limoncillo, hojas de naranjo agrio, ciruelo y guanábano, con las que la gestante se baña nueve días antes de la fecha que el médico estima para su parto. Se hacen en la noche antes de dormir y el agua debe limpiar el cuerpo de abajo hacia arriba, hasta la cintura. Tienen como finalidad facilitar el momento del parto y limpiar el feto.

$A$ veces son todas gordas y uno cree que va a tener un bebé muy grande y mentiras que no, es el frío el que las infla más, porque a veces el bebé es una cosita de nada y ellas gordas, por eso son buenos los baños. (Mariela)

Mi mamá siempre me daba agua con canela en las mañanas para que no aguantara frío. Porque el niño mantenía mucho hipo y me decían que era porque mantenía la barriga muy mojada. (Lucía)

La bebida de mayor uso es el agua de canela que, en algunos casos, se toma durante todo el embarazo para eliminar los fríos que puedan invadir el vientre de la madre y, en otros, en los instantes próximos al parto para apresurar el nacimiento. Al agua con canela se le adiciona yerbabuena o aguardiente y se cocina, como todas las infusiones, con panela.

Actividades durante la gestación. La gestación transcurre como una trayectoria corporal y social que se hace en compañía de familiares y personas cercanas. Las conversaciones y actividades cotidianas giran en torno a los cuidados de la gestante y el bebé, los roles de una madre, las pautas de crianza y el cuidado de los hijos, que tienen la doble intencionalidad de compartir consejos para el mantenimiento y el mejoramiento de la salud, y reproducir las lecciones de género alrededor de la maternidad. 
En el momento en que se confirma el embarazo, hay una serie de consideraciones respecto a las labores cotidianas, entre ellas, evitar esfuerzos excesivos, adicionar y restringir ciertos alimentos y evitar la exposición al medio, como cubrirse de la lluvia, el viento, la luna llena y los eclipses.

Las labores cotidianas escasamente pueden interrumpirse a causa de la gestación; no obstante, se hacen algunas consideraciones que dependen del tipo de trabajo y las condiciones de clase de las mujeres. Quienes se dedican al mantenimiento de la casa y a la alimentación de la familia continúan atendiendo a los menores (hijos, nietos, sobrinos) y a los hombres que habitan la unidad doméstica, con posibilidad de algunos momentos de receso. Las mujeres que trabajan en alimentaderos ${ }^{4}$ se desenvuelven simultáneamente en las labores productivas para el hogar y el cuidado de su familia, ocupándose de cocinar, servir y limpiar, sin que la maternidad les permita alguna tregua. Sin embargo, no cuidar del equilibrio entre lo frío y lo caliente durante la gestación es percibido como tanto o más peligroso que el esfuerzo físico.

Ella se cuidaba en el embarazo, claro que en este no se cuidó, en el de Estefanía, porque ella salía de la cocina y se ponía a lavar ahí mismo y pues con eso coge mucho frío. De eso yo creo que la niña quedó sufriendo neumonía; recibía el frío. El calor de la cocina y de ahí se va a lavar, pues recibe frío. (Mariela)
Aquellas que se dedican a la recolección de café cerca de sus viviendas, en pequeñas parcelas, o que trabajan en alguna de las haciendas de la región, continúan cumpliendo con su trabajo durante la mayoría del tiempo de gestación $y$, si la solvencia de su grupo familiar lo permite, ponen mayor esmero en su cuidado durante los últimos días. También es común que, por temporadas, algunas mujeres viajen a las ciudades capitales aledañas a trabajar en labores de aseo en hogares de clase media o alta; presionadas por la necesidad de conseguir el sustento económico para sus hijos, que difícilmente se logra en el municipio cuando no es tiempo de cosecha.

\section{Promotoras de salud}

En el ámbito biomédico, las prácticas de cuidado y atención más destacadas son los controles prenatales y la ecografía, que en esta comunidad están mediadas por la promotora rural de salud, quien hace la remisión a los controles durante las visitas domiciliarias.

La promotora es un brazo de la atención hospitalaria que llega hasta el espacio doméstico, compartiendo los códigos y los modos de interacción de las mujeres de la comunidad. La promotora ostenta un estatus de autoridad en la vereda, pero su cercanía con las familias hace que establezca con ellas un tipo de relación sustancialmente distinta a la de los profesionales de salud en el hospital. La visita de la promotora transcurre con la informalidad característica de los encuentros entre vecinas o conocidas.

${ }^{4}$ Lugares en los que se ocupan de la manutención de las trabajadoras y trabajadores que llegan de otras zonas del país en épocas de cosecha o en otros momentos del año para emplearse temporalmente en alguna labor relacionada con el cultivo de café. 
Les tomo la presión, miro si se le están hinchando los pies o las manos, algún síntoma, que les duele la cabeza, visión borrosa, a ver cómo se han sentido ellas en el embarazo. $Y$ ya de acuerdo a lo que uno ve les da las enseñanzas: si están tomando lo que les manda el médico, qué alimentación tienen, que si dan caminaditas, todos esos consejitos que uno les da. (Verónica)

Siempre se recuerdan las instrucciones relativas a síntomas y signos de alarma, alimentación, actividad física y estimulación intrauterina. Pero el encuentro no se refiere exclusivamente al cuidado de la salud, se transmiten también instrucciones sociales sobre la vivencia de la maternidad, que incluyen la insistencia de actitudes afectuosas de la madre hacia el feto; las promotoras suelen hablar de la importancia de "esperar a los hijos con amor".

¿Y si está contenta con su bebé? ¿Si le habla? ¿si le canta? [...], todo eso es muy importante, porque la personalidad del niño se va formando desde el embarazo, todo lo que uno siente el bebé se da cuenta. Por eso es tan importante que el niño sea deseado, son niños que van a tener una buena personalidad, son niños sin problemas. (Ximena)

En estas visitas, las mujeres encuentran un espacio distinto al de las relaciones jerárquicas que operan en el hospital para resolver inquietudes sobre su proceso de gestación y ampliar la información que les es dada por médicos y enfermeras. Estas visitas sirven de mediadoras entre baños, bebidas o alimentos fríos y calientes, y temas sobre alimentación saludable, síntomas de riesgos, medicamentos y suplementos vitamínicos. Adicionalmente, las promotoras asesoran a las mujeres sobre los trámites burocráticos para acceder a los servicios de salud, incluyendo una ficha de remisión para el control prenatal, que busca garantizar la asistencia periódica al hospital.

\section{Atención hospitalaria}

La población rural que participó en este estudio asiste al hospital público de primer nivel, ubicado en el casco urbano del municipio. Allí se atienden los controles prenatales y los partos de bajo riesgo. Además de los chequeos médicos, uno de los principales componentes de los controles prenatales es la advertencia sobre los riesgos del embarazo, las señales de alarma y los hábitos necesarios para prevenir complicaciones. Pese a la distancia que los profesionales de salud intentan tomar frente a los saberes populares, existe una constante interacción entre estos últimos y el saber biomédico, tanto en el ámbito doméstico como en el hospitalario. Las recomendaciones de los profesionales se discuten en la esfera doméstica, en donde se ponen en consideración con otros saberes alrededor de la maternidad que les son transmitidos por madres y abuelas. Las sugerencias dietarias de los profesionales de salud, por ejemplo, siempre se revisan bajo la lógica de los alimentos fríos y calientes.

El espacio hospitalario tampoco es ajeno a los saberes que las mujeres comparten en sus redes cercanas. Las infusiones, ungüentos y brebajes calientes rondan sutilmente las consultas médicas, las salas de espera y los pasillos de las instituciones de salud. 
Allí las mujeres se recomiendan unas a otras diferentes prácticas de cuidado y, en ocasiones, médicas/os y enfermeras aconsejan confidencialmente las recetas que han conocido en otros espacios o que les han sido mencionadas por otras pacientes. Paradójicamente, una consulta hospitalaria puede llegar a convertirse en difusora de los saberes populares de los que tanto busca diferenciarse la medicina occidental.

La interacción de estos saberes, así como las contradicciones que acarrea, también se refleja en las respuestas de los profesionales de salud:

Yo tengo que respetar que hay un médico, que estudió seis años, que él maneja medicina y no botánica; pero yo soy una persona muy creyente en las plantas. El alóe vera, por ejemplo. Aquí las mujeres usan muchas plantas, aunque no siempre es recomendable. (Soledad, enfermera)

La siguiente cita ilustra las tensiones entre estos saberes y la influencia inevitable del contexto cultural:

Se escucha mucho el de tomar agüita de la hoja de brevo o bañarse con brevo, entonces, ¿qué es lo que hace?: una retención placentaria. Esa es la complicación. Cuando las viejas nos dicen: "es que yo tomé agüita de brevo", nos provoca estriparlas. Eso es respetable, pero mejor que no lo hagan. (Carolina, médica).

No obstante, la misma médica fue cuidada por su madre durante su gestación y puerperio:
A mi me cuidaron la dieta de los cuarenta días. En la primera y segunda semana no me dejaban ver televisión, ni leer, ni nada que porque se me agotaba la vista, que yo no sé qué. Me bañaban en la cama con unas yerbas con aguas. $Y \mathrm{mi}$ mamá me decía: "esto es lo que le va a dar salud y vida más adelante". Pero la dieta de los cuarenta días es algo hasta chistoso, pero si ella lo dice es respetable. $Y$ yo ya lo viví. (Carolina, médica)

Desde las campañas de institucionalización del parto, lideradas por las primeras promotoras de salud de la zona, los controles prenatales se establecieron como componente indispensable del cuidado de la maternidad y la asistencia a terapeutas no biomédicos se volvió cada vez menos frecuente. No obstante, cuando existen barreras en el acceso a la atención hospitalaria, algunas mujeres acuden a sanadores por fuera del sistema institucional de salud.
[...] No era capaz de voltearme ni nada, entonces me iba a ir pa' el hospital, pero cuando eso estaban en paro, entonces no me aten- dieron allá y me fui pa' donde un señor que sabía mucho, con solo mirar la mano él ya sabía qué era lo que uno tenía... pues yo des- esperada por ese dolor que yo no aguantaba nada. (Rosa)

Por el contrario, en el parto el saber biomédico opera como el principal y, con frecuencia, único conocimiento autorizado. El hospital de este municipio ha transitado por repetidas crisis económicas desde su transformación en Empresa Social del Estado (ESE). No 
obstante, las mujeres no cuestionan la superioridad del parto institucionalizado frente al parto en sus viviendas o al atendido por terapeutas no biomédicos. Como resultado de las campañas de salud pública desde la década de los 60 y la consecuente estigmatización de las parteras, otras alternativas de parto se consideran peligrosas y primitivas. Quienes han dado a luz en casa son mujeres mayores de 50 años, que tienen o tenían en su familia cercana una partera, o bien, mujeres provenientes de otras zonas rurales del país, en donde las posibilidades de acceso al hospital de la cabecera municipal son muy pocas.

\section{Retorno al ámbito doméstico en el posparto}

El posparto es un tiempo de cuidado exclusivo del entorno doméstico. El cuerpo femenino debe retornar a su equilibrio natural a través de estrictos procedimientos de restitución que le permitirán expulsar los fríos a los que haya sido susceptible durante la gestación. Los cuarenta días que transcurren después del parto, conocidos como "la dieta", son un tiempo de restitución biológica y social en el que la mujer y su familia retoman el control sobre el cuidado e inicia una recuperación circunscrita, exclusivamente, al espacio doméstico.

Yo la cuidaba [a mi hija], la cuidaba de que no se le entraran los vientos, de que se entrara temprano, que no se serenara, que no recibiera la luna, ni los vientos de agua. Los vientos así de agua, disque eso es malo, uno coge unos dolores y eso no son capaces de cogerlo los médicos; eso es como una noralgia que le da a uno, como un viento que se le arrejunta a uno en la carne y los médicos no conocen eso, así vaya uno con los dolores no los curan, porque eso es como un viento. Mi papá decía que, si uno se desmandaba con una dieta, eso no lo curan los médicos, así se tome uno radiografías, porque esos son vientos de dieta. Papá decía que para curarse tiene que tener otro hijo y cuidarse uno bien cuidado. (Celeste)

Durante el posparto, buena parte de las atenciones van dirigidas a proteger a la mujer de los fríos, vientos o aires que podrían amenazar el restablecimiento del equilibrio térmico. Por ello, durante esos días la mujer debe permanecer en casa y en reposo con su bebé. Es entonces cuando se le practican sahumerios y limpiezas con plantas aromáticas de naturaleza caliente, destinados a expulsar el frío y las impurezas del cuerpo. El último de los cuarenta días, cuando se "cumple la dieta", es definitivo en la reincorporación de la madre a su rutina diaria, el cuerpo se purifica y son eliminados todos los fríos que pudiesen haber entrado desde el embarazo hasta ese momento. Al sahumerio con ramas calientes y el chocolate se le han sumado purgantes que anteriormente se preparaban con plantas, ahora se compran en las tiendas naturistas del pueblo.

\section{Discusión}

Algunos estudios de Terapia Ocupacional han descrito la maternidad como una ocupación humana que involucra una amplia gama de habilidades y actividades que marcan el paso a la vida adulta (Farber, 2000; Maceira-Domínguez, Rivas-Quarneti, \& García-Gonza- 
lo, 2018); otros han priorizado la idea de la maternidad como rol social que modifica las ocupaciones de las mujeres en los diferentes ámbitos de la vida (Horne, Corr \& Earle, 2005); unos más han descrito la centralidad del rol de las madres en los procesos de cuidado y rehabilitación (Moreno \& Salas, 2017). Desde todas las perspectivas, la maternidad es reconocida como una experiencia vital que encierra significados profundos, los cuales se comparten socialmente. En ese sentido, este artículo propone una mirada etnográfica sobre la gestación y el puerperio como experiencias que involucran actividades cotidianas de cuidado y significados alrededor de la maternidad, que transcurren y se comparten en los ámbitos doméstico y comunitario. La mirada etnográfica ofrece luces para abordar la salud materna desde un enfoque comunitario que reconozca la manera en que se construyen sentidos intersubjetivos sobre salud y bienestar. Si bien la salud materna es aún un campo por explorar para la Terapia Ocupacional, es precisamente en la particularidad de las experiencias corporales y los sentidos de vida de los sujetos en donde esta profesión puede hacer importantes aportes para acompañar la maternidad como proceso socio-biológico.

Las narrativas de las mujeres señalan el cuidado en sus comunidades, la atención hospitalaria y la visita de las promotoras de salud como los ejes centrales de cuidado. Siguiendo la propuesta conceptual de inclusión sociocomunitaria de Pino y Ceballos, (2015), puede sugerirse que, desde el ámbito doméstico y comunitario, las mujeres son incluidas en un universo de sentido alrededor de su cuerpo, la relación con el entorno y la maternidad; y, desde el ámbito hospi- talario, ellas participan como ciudadanas de un sistema de salud regido por el saber biomédico.

La lógica de frío y caliente, así como otros saberes populares sobre la maternidad, serían componentes de lo que Pino y Ceballos (2015), siguiendo planteamientos fenomenológicos, denominan "el mundo de la vida". Este mundo se refiere a la experiencia cotidiana, al sentido común y a los significados sobre la vida, la relación con otros y con la naturaleza. La inclusión comunitaria, por tanto, consiste en la participación en esta esfera de sentido. La atención hospitalaria, por otro lado, consiste en la incorporación de lo que estas autoras Ilaman el mundo del sistema. Este mundo, que para el caso del presente estudio incluyó controles prenatales, ecografías obstétricas y atención al parto, consiste en la estructura institucionalizada en la cual las mujeres participan en condición de pacientes. El mundo del sistema está regido por una jerarquía preestablecida en relación con la cercanía al saber biomédico, tiene un alto nivel de formalización y estandarización de los procedimientos, y funciona bajo lógicas burocráticas de garantía de derechos. No obstante, la inclusión social, que consiste en la inclusión en este sistema, requiere en esta comunidad de una intermediadora: la promotora de salud, quien reconoce la importancia del mundo de la vida y del mundo del sistema, genera traducciones de sentido y señala rutas de acceso al sistema de salud.

Entre las mujeres que participaron en este estudio, las nociones de frío y caliente son un componente fundamental del universo de sentido de la gestación y el puerperio. El equilibrio entre estas dos propiedades alude a percepciones 
y representaciones de la corporalidad en relación con el medio ambiente, que están en línea con la idea de vida de las comunidades que trabajan la tierra. Según Motte- Florac (2008), los procesos de compensación entre lo frío y lo caliente adquieren una importancia destacada en la etiología de las enfermedades en poblaciones agricultoras, en donde la influencia del clima en las cosechas compromete directamente el sustento y la vida de la comunidad, "el frío, el viento, el calor, forman parte de las marcas ineludibles de la enfermedad, así como el granizo, las heladas y la tormenta lo son de la desgracia" (Motte-Florac, 2008, p.181).

Las nociones de frío y caliente encontradas en este estudio guardan numerosas coincidencias con las que se han descrito en otras zonas del país y de Latinoamérica (Katz, 1992; Chávez-Hualpa, 1996; Rojas-Trejos, 2000). No obstante, es destacable que, a diferencia de otras poblaciones andinas, en la comunidad que aquí se describe, el equilibrio entre lo frío y lo caliente está relacionado casi exclusivamente con la maternidad. Probablemente, el carácter doméstico y comunitario de la experiencia de la maternidad ha permitido la reproducción de estos saberes al margen de la influencia del saber biomédico.

Este mundo de sentido se construye cotidianamente a través de redes menos jerárquicas e institucionalizadas que en el mundo del sistema. Los saberes y prácticas de cuidado circulan en las visitas entre familias, en el mercado, en la plaza o en el transporte entre el pueblo y las veredas, y todos están autorizados para hacer recomendaciones. Las personas se comparten recetas, se regalan plantas medicinales, se aconsejan consumir o evitar alimentos, ofrecen explicaciones sobre el malestar en espacios públicos y privados, sin establecer una jerarquía rígida en la que unos sujetos están del lado de la verdad y otros de la creencia.

Si bien el mundo del sistema, que aquí relacionamos con el sistema de salud, tiene estructuras y procesos mucho más rígidos, también se ha ido transformando a raíz de los cambios en el contexto. Los programas de salud materno-infantil, desde la década de los 60 en esta región, implementaron elementos que ahora son centrales en el proceso de cuidado de la maternidad, tales como la visita de las promotoras de salud, las citas de control prenatal y el parto hospitalario. Asimismo, la transición del sistema de salud en la década de los 90, que reemplazó el modelo de financiamiento de los hospitales públicos, de subsidio a la oferta por subsidio a la demanda, convirtió la gestación, el parto y el puerperio en trayectorias burocráticas y administrativas que delinean los ejercicios de ciudadanía característicos de un sistema de salud basado en la atención gerenciada (Abadía y Oviedo, 2009).

No obstante, el mundo del sistema no es ajeno a la configuración de sentido propio del mundo de la vida. No solamente el saber biomédico juega un papel importante en la manera en que las mujeres entienden la maternidad como experiencia corporal y social, sino que los saberes populares transgreden los espacios institucionalizados de la biomedicina. La atención hospitalaria tiene lugar en contextos socioculturales particulares, en los que operan distintas lógicas sobre la vida, y funciona a través de integrantes de esa misma comunidad. El personal de 
salud ocupa también los roles de madre, hijo, tía, y como tales se involucran en esas otras redes de conocimiento. Los saberes sobre plantas y alimentos fríos y calientes rondan sutilmente las consultas médicas. Por un lado, las salas de espera y los pasillos de las instituciones de salud son también espacios comunitarios y, por otro, los profesionales de salud reconocen el valor de los saberes populares en sus espacios domésticos.

La participación de las mujeres en el mundo de la vida y en el mundo del sistema conecta significados, elementos terapéuticos y mecanismos de atención que se sincretizan en el devenir de las actividades cotidianas, dando lugar a un proceso que no solo mejora y mantiene el bienestar de la madre, sino que también constituye y redimensiona su cuerpo continuamente. El análisis de procesos de salud-enfermedad, como experiencias de vida situadas en contextos socioculturales específicos, nos permite avanzar hacia modelos comunitarios que identifiquen los problemas de los sujetos y las comunidades en la vida cotidiana, sean flexibles a los contextos de implementación y desarrollen abordajes interdisciplinarios (Palacios, 2017).

\section{Conclusión}

La Terapia Ocupacional tiene el privilegio de acompañar a los sujetos más allá de los ámbitos biomédicos, para ayudarles a participar en el mundo de la manera en que mejor pueden hacerlo. En ese sentido, este artículo responde a la invitación de pensar en lo cotidiano como posibilidad de entender tanto las construcciones intersubjetivas de salud y bienestar de las madres, como la manera en que se ar- ticulan diferentes sectores de cuidado en un contexto sociocultural particular. Esta exploración de lo cotidiano abre posibilidades a enfoques comunitarios que reconozcan los significados sobre el cuerpo y la vida, más allá de intervenciones jerárquicas y exclusivamente técnicas.

Escuchar las experiencias de las mujeres que participaron en este estudio permitió acceder a esas realidades de cuidado que se construyen en el ámbito doméstico y comunitario alrededor del cuerpo, la salud y la maternidad, que surgen y se transforman en los encuentros y desencuentros entre los distintos saberes, estableciendo prácticas, significados y representaciones. La mirada etnográfica no solo contribuye a un análisis de lo cotidiano, sino también del tejido social en la experiencia de los individuos. Entre las limitaciones de esta investigación se encuentra la imposibilidad de generalizar los resultados a otras comunidades campesinas. Se recomienda que futuros estudios involucren metodologías mixtas (cuantitativa y cualitativa) y desarrollen análisis comparados entre diferentes comunidades.

\section{Referencias}

Acharya, V. (2014). Preparing for motherhood: a role for occupational therapy. World Federation of Occupational Therapists Bulletin, 70(1), 16-17. doi: https://doi.org/10.1179/ otb.2014.70.1.005

Abadía, C. E., y Oviedo, D. G. (2009). Bureaucratic Itineraries in Colombia. A theoretical and methodological tool to assess managed-care health 
care system. Social Science and Medicine, 68, 1163-1160.

Arellano, V.M. \& Vega, M.C. (2005). Implementación de la intervención temprana con mujeres embarazadas y sus hijos desde el nacimiento hasta los seis meses de vida. II Parte. Revista Chilena de Terapia Ocupacional, 9, 49-66.

Blázquez, M. I. (2005). Aproximación a la antropología de la reproducción. Revista de Antropología Iberoamericana, 42, 1-25.

Chávez Hualpa, F. (1996). Mujeres que curan, mujeres que creen: un perfil de la medicina femenina. En M. Polia, Despierta, remedio, cuenta--: adivinos y médicos del Ande. Lima: Pontificia Universidad Católica del Perú, Fondo Editorial.

Farber, R.S. (2000). Mothers with disabilities: in their own voice. American Journal of Occupational Therapy, 54(3), 260-268.

Fernandes, J. (2018). Occupational Therapists' Role in Perinatal Care: A Health Promotion Approach. The American Journal of Occupational Therapy, 72 (5), 1-4. doi: https://doi.org/10.5014/ ajot.2018.028126

García Ruiz, S. (2017). El arte de escribir sobre lo cotidiano. Revista Ocupación Humana, 17(2), 3-4. Doi: https:// doi.org/10.25214/25907816.167

Gee, B., Kunkel, S., Troy, L., Swann, H., Devine, N., Burgett, N., ... \& RamsdeII-Hudock, H. (2017). Infant and Maternal Reciprocity as Expressed Through Play Performance and Participation. American Journal of Occupational Therapy, 71(4Sup.1). doi: doi:10.5014/ ajot.2017.71S1-PO2089
Ginsburg, F., \& Rapp, R. (1995). Conceiving the New World Order: The Global Politics of Reproduction. Berkeley, CA: University of California Press.

Horne, J; Corr, S; Earle, S. (2005) Becoming a Mother: Occupational Change in First Time Motherhood. Journal of Occupational Science, 12(3), 176183. doi: https://doi.org/10.1080/1442 7591.2005 .9686561

Katz, E. (1992). Del frío al exceso de calor: dieta alimenticia y salud en la Mixteca. En P. Sesia-Lewis (Ed.), Medicina traducional, herbolaria y salud comunitaria en Oaxaca (pp. 99113). Oaxaca: CIESAS.

Maceira-Domínguez, M., Rivas-Quarneti, N., García-Gonzalo, N. (2018). "Yo lo parí y él me dio la vida": estudio de la transición ocupacional vinculada a la maternidad de dos mujeres con trastorno mental. Cadernos Brasileiros de Terapia Ocupacional, 26(2), 271-285. doi: https://doi.org/10.4322/2526-8910. ctoAO1156

Moreno, R., \& Salas, M. (2017). Sabidurías de una madre. Relatos de una recuperación. Revista Chilena de Terapia Ocupacional, 17(1), 165-169. doi: 10.5354/0719-5346.2017.46389

Morgan, L. M., \& Roberts, E. F. S. (2012). Reproductive governance in Latin America. Anthropology \& Medicine, 19(2), 241-254. https://doi.org/10.108 0/13648470.2012.675046

Motte-Florac, E. (2008). Santos, humores y tiempo: el clima y la salud entre los purépechas de la sierra tarasca (Michoacán). En A. Lammel, M. Goloubinoff, \& E. Katz (Eds.), Aires y lluvias. 
Antropología del clima en México (pp. 179-210). México: CEMCA-CIESAS-IRD.

Palacios, M. (2017). Reflexiones sobre las prácticas comunitarias: aproximación a una Terapia Ocupacional del Sur. Revista Ocupación Humana, 17 (1), 73-88.

Pino, J. \& Ceballos, M. (2015). Terapia ocupacional comunitaria y rehabilitación basada en la comunidad: hacia una inclusión sociocomunitaria. Revista Chilena de Terapia Ocupacional, 15(2). doi: 10.5354/0719-5346.2015.38167

Rodríguez-Hernández, M., Flores-Martos, J. A. \& Moruno-Miralles, P. (2012). Medicalización en las mujeres embarazadas y parturientas: una aproximación desde Terapia Ocupacional. Parte I. Revista Terapia Ocupacional Galicia, 9(15), 1-18.

Rojas-Trejos, B. (2000). Cuerpos tiernos y abiertos. Embarazo y parto entre las mujeres campesinas de Mucuchies. Boletín Antropológico, (49), 75-92.

Rubio-Grillo, M.H., Perdomo-Oliver, N.M. \& Zamundio-Espinosa, D.C. (2018). Estimulación prenatal en gestantes de alto riesgo obstétrico: una mirada desde la ocupación. Aquichan, 18(4), 426-437. doi: http://dx. doi.org/10.5294/aqui.2018.18.4.5

Trovatti, T. V. \& Peréz Martínez, S. (2012). La actuación del terapeuta ocupacional en el embarazo, parto y puerperio : un camino por explorar. Reduca, 4(11), 1-17.

Visser, M., Nel, M., la Cock, T., Labuschagne, N., Lindeque, W., Malan, A. \& Viljoen, C. (2016). Breastfeeding among mothers in the public health sector: the role of the occupational therapist. South African Journal of Occupational Therapy, 46(2), 65-72. doi: https://dx.doi.org/10.17159/23103833/2016/v46n2a11 